\title{
A SINGLE LOCUS MASS-ACTION MODEL OF ASSORTATIVE MATING, WITH COMMENTS ON THE PROCESS OF SPECIATION
}

\author{
WILLIAM S. MOORE \\ Department of Biology, Woyne Stote University, Detroit, \\ Michigan 48202, U.S.A.
}

Received 13.ix.78

\begin{abstract}
SUMmary
Genes that cause positive assortative mating have the potential of effecting reproductive isolation and hence speciation. A one-locus-2-allele model of assortative mating is investigated. In this model, when two individuals encounter, they mate with probability $1, \alpha$ or $\beta$ depending on whether they share 2,1 or 0 alleles respectively at the assortative mating locus. The special case where $\alpha=0.5 ; \beta=0$ is investigated extensively. Assortative mating eliminates genetic polymorphisms. The only non-trivial equilibrium occurs when each homozygote has a frequency of 0.5 and there are no heterozygotes, but this equilibrium is unstable. Numerical analysis suggests that this is also true when assortment is only partial $(\beta>0)$. When the alleles are allowed to mutate from one form to the other, a stable non-trivial equilibrium results, but one allele or the other is very rare. When the alleles affect fitness in some additional way, the assortative mating locus will be polymorphic provided there is substantial hybrid superiority; e.g. when the homozygotes are equally fit, the heterozygote must be approximately twice as fit. Similarly, favourable mutants at the assortative mating locus cannot enter a population unless they enhance the fitness of both their genotypes rather substantially. Thus, in the hypothesis of speciation where premating isolating mechanisms are supposed to evolve as a response to selection against hybrids, there is some doubt as to whether genetic variation for assortative mating would exist, and, if it did, whether it would always respond to selection.
\end{abstract}

\section{INTRODUGTION}

INTERBREEDING of closely related sympatric species is often prevented by premating isolating mechanisms (Mayr, 1963); i.e. by some form of positive assortative mating within each species. The sequence of events usually postulated for this process of speciation includes an episode of selection against hybrids formed from distinctly coadapted gene pools. The hybridisation episode might result from secondary contact following geographical isolation (Remington, 1968) or, alternatively, might involve elements of a continuous population that have diverged along an environmental gradient (Endler, 1977). In either case, selection should favour those individuals of "pure" phenotype who prefer to mate with their own kind because they would not waste reproductive energy on maladapted hybrid offspring. It is reasonable to suppose that such a selection regime would reorganise variation at assortative mating loci in such a way that premating reproductive isolation would evolve; i.e. speciation would occur (Dobzhansky, 1940; Huxley, 1942; Mayr, 1963). The mathematical model presented in this paper has raised some doubt in my mind as to whether gene loci that cause 
positive assortative mating would usually be polymorphic, and, if they were, whether they would respond to such selection pressures. The model is simple; nonetheless, it provides some insight into the population genetics of assortative mating, and, at this juncture, it is not certain that more realistic and, hence, more complex systems would behave in an essentially different manner.

The situation modelled is as follows: individuals encounter one another at random, and, given that two individuals have encountered, they mate with probability $1, \alpha$ or $\beta$ depending on whether they share 2,1 or 0 identical alleles $\left(A, A^{\prime}\right)$ at a single locus which affects mate preference. This assortative mating scheme is illustrated in table 1 . The organisms are hermaphroditic or, if sexes are separate, there is a $1: 1$ sex ratio, and the assortative mating alleles are expressed identically in both sexes. The number of encounters is assumed to be finite and independent of whether or not mating occurs. The population is assumed to be very large. Generations are discrete.

TABLE 1

The probabilities of various matings, given that two genotypes have encountered

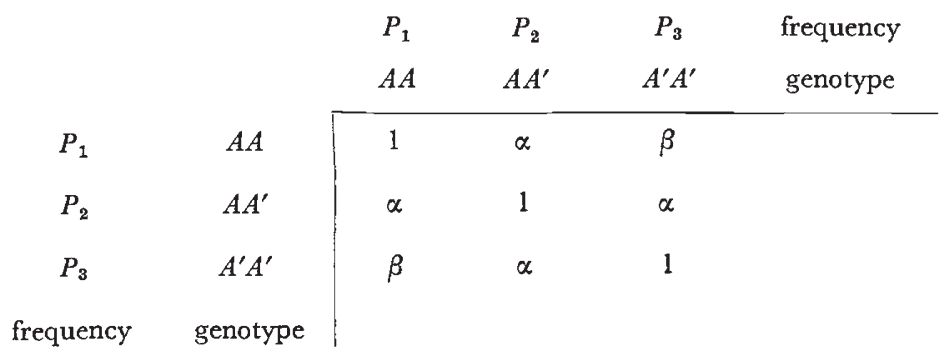

The term encounter has a somewhat expanded meaning as used here. As in its normal usage, one can envisage two animals roaming about in a habitat and actually encountering in a physical sense. The two animals would then decide whether to mate based on their phenotypic similarity. In the expanded sense, to encounter could, as well, mean that two flowering plants are separated by considerable distance but have sufficient proximity that it is possible for a pollinator to fly from one to the other thereby allowing the two to mate if they are of appropriate genotypes.

As a realistic, albeit hypothetical, example of a mating system that might be reflected by this model, consider pollination in Lythrum salicaria (purple loosetrife). L. salicaria is a tall perennial $(0.5-2 \mathrm{~m})$ with an inflorescence formed as a long terminal spike. The flowers are tristylous and self-incompatible; pollination, thus, depends upon insect vectors. Levin and Kerster (1973) reported that the correlation between stature (inflorescence height) of mating plants was $0 \cdot 86$. This strong correlation results from a penchant in hive-bees (Apis mellifera) to forage in a horizontal plane. Thus, despite substantial heterogeneity in inflorescence height $(1.02-1.91 \mathrm{~m})$ in the population studied by Levin and Kerster, 80 per cent of the pollinations of a given inflorescence were effected by bees coming from an inflorescence whose height differed by less than $0.064 \mathrm{~m}$. Certainly a substantial amount of this heterogeneity in stature would be environmentally induced; however, plant stature is obviously under some genetic 
control, and, as a consequence, a gene that affects stature might establish assortative mating.

The model presented here was originally formulated by Karlin and Scudo (1969, pp. 507-508) as one of several assortative mating models presented in tandem papers (Scudo and Karlin, 1969 is the other paper). Their analysis, however, was restricted to the single conclusion that this situation leads to an unstable equilibrium. Karlin and Scudo (1969) described this model as a symmetrical sex mass action model because the alleles were identically expressed in the two sexes and because of the analogy of the dynamics of the population to the dynamics of the elastic collisions of particles. Henceforth, I will refer to the present model as the symmetricalsex-mass-action model or simply the mass-action model.

The purpose of the present paper is to extend the analysis of the massaction model; specifically, I will consider assortative mating alone, assortative mating and mutation, assortative mating and selection, and, finally, the combined effects of all three. In so doing, I will focus on those aspects of the model pertinent to the process of speciation.

\section{Assortative mating}

The kinds of matings and expected ratios of offspring genotypes are tabulated in table 2. The frequencies of the three genotypes that will comprise the next generation $\left(P_{1}^{\prime}, P_{2}^{\prime}\right.$, and $\left.P_{3}^{\prime}\right)$ are given in terms of their frequencies in the previous generation $\left(P_{1}, P_{2}\right.$ and $\left.P_{3}\right)$. These were obtained by summing the values in each offspring column and dividing by a normalising factor $\bar{N}$. The normalising factor was chosen such that $P_{1}^{\prime}+P_{2}^{\prime}+P_{3}^{\prime}=1$ and is simply the sum of values in the offspring portion of the table. $P_{3}$ can be eliminated from the expression for $\bar{N}$ by substituting $\left(1-P_{1}-P_{2}\right)$ for $P_{3}$. A pair of simultaneous difference equations giving the change in frequencies of genotypes $A A$ and $A A^{\prime}$ in one generation $\left(\Delta P_{1}\right.$ and $\Delta P_{2}$ ) can now be formulated from the values given in table 1 .

TABLE 2

Frequencies of offspring genotypes $\left(\mathbf{P}_{1}^{\prime}, \mathbf{P}_{2}^{\prime}\right.$ and $\left.\mathbf{P}_{3}^{\prime}\right)$ in the next generation resulting from assortative mating

\begin{tabular}{|c|c|c|c|c|c|}
\hline \multirow[b]{2}{*}{ Mating } & \multirow{2}{*}{$\begin{array}{l}\text { Probability } \\
\text { of encounter }\end{array}$} & \multirow{2}{*}{$\begin{array}{l}\text { Probability } \\
\text { of mating given } \\
\text { an encounter }\end{array}$} & \multicolumn{3}{|c|}{ Offspring } \\
\hline & & & $A A$ & $A A^{\prime}$ & $A^{\prime} A^{\prime}$ \\
\hline $\begin{array}{l}A A \times A A \\
A A \times A A^{\prime} \\
A A \times A^{\prime} A^{\prime} \\
A A^{\prime} \times A A^{\prime} \\
A A^{\prime} \times A^{\prime} A^{\prime} \\
A^{\prime} A^{\prime} \times A^{\prime} A^{\prime}\end{array}$ & $\begin{array}{c}P_{1}^{2} \\
2 P_{1} P_{2} \\
2 P_{1} P_{3} \\
P_{2}^{2} \\
2 P_{2} P_{3} \\
P_{3}^{2}\end{array}$ & $\begin{array}{l}1 \\
\alpha \\
\beta \\
1 \\
\alpha \\
1\end{array}$ & $\begin{array}{c}P_{2}^{2} \\
\alpha P_{1} P_{2} \\
0 \\
\frac{1}{4} P_{2}^{2} \\
0 \\
0\end{array}$ & $\begin{array}{c}0 \\
\alpha P_{1} P_{2} \\
2 \beta P_{1} P_{3} \\
\frac{1}{2} P_{2}^{2} \\
\alpha P_{2} P_{3} \\
0\end{array}$ & $\begin{array}{c}0 \\
0 \\
0 \\
1 P_{2}^{2} \\
\alpha P_{2}^{2} P_{2} \\
P_{3}^{2}\end{array}$ \\
\hline
\end{tabular}

Genotypic frequencies in the next generation:

$P_{1}^{\prime}=\frac{P_{2}^{2}+\alpha P_{1} P_{2}+\frac{1}{4} P_{2}^{2}}{\bar{N}}$

(1) $\quad P_{2}^{\prime}=\frac{\alpha P_{1} P_{2}+2 \beta P_{1} P_{3}+\frac{1}{2} P_{2}^{2}+\alpha P_{2} P_{8}}{\bar{N}}$

(2) $\quad P_{8}^{\prime}=\frac{\frac{1}{4} P_{2}^{2}+\alpha P_{2} P_{3}+P_{3}^{2}}{\bar{N}}$

where:

$$
\begin{aligned}
& P_{1}+P_{2}+P_{3}=1 \quad(4) \\
& \tilde{N}=1+2\left[(1-\beta) P_{1}^{2}+(1-\alpha) P_{2}^{2}+(1-\beta) P_{1} P_{2}+(\beta-1) P_{1}+(\alpha-1) P_{2}\right]
\end{aligned}
$$




$$
\begin{gathered}
\Delta P_{1}=P_{1}^{\prime}-P_{1}=\left(P_{1}^{2}+\alpha P_{1} P_{2}+\frac{1}{4} P_{2}^{2}-\bar{N} P_{1}\right) / \bar{N} \\
\Delta P_{2}=P_{2}^{\prime}-P_{2}=\left[2 \beta P_{1}\left(1-P_{1}-P_{2}\right)+\frac{1}{2} P_{2}^{2}+\alpha P_{2}\left(1-P_{2}\right)-\bar{N} P_{2}\right] / \bar{N}
\end{gathered}
$$

Again $P_{3}$ has been eliminated by $P_{3}=1-P_{1}-P_{2}$.

Although these general difference equations have proved intractable to me, the equilibrium conditions for the special case $\beta=0$ can be obtained, and, indeed, this is the most interesting case because $\beta=0$ leads to speciation if the heterozygotes, $A A^{\prime}$, are eliminated from the population. If equilibria exist, then $\Delta P_{1}=\Delta P_{2}=0$ at the corresponding equilibrium values, $\hat{P}_{1}$ and $\hat{P}_{2}$, of the two genotypes. Thus, setting equations (6) and (7) equal to zero yields simultaneous algebraic equations whose solutions provide potential equilibrium conditions:

$$
\begin{aligned}
& \hat{P}_{1}^{2}+\alpha \widehat{P}_{1} \widehat{P}_{2}+\frac{1}{4} \hat{P}_{2}^{2}-\bar{N} \hat{P}_{1}=0 \\
& \frac{1}{2} \hat{P}_{2}^{2}+\alpha \hat{P}_{2}\left(1-P_{2}\right)-\bar{N} \hat{P}_{2}=0 .
\end{aligned}
$$

It is immediately obvious that $\hat{P}_{2}=0$ would satisfy equation $(9)$, and substituting $\hat{P}_{2}=0$ into equation (8) yields the equilibrium solution set given in table 3 . However, it is also apparent that if $\bar{N}=\frac{1}{2} \hat{P}_{2}+\alpha\left(1-\hat{P}_{2}\right)$, then equation (9) would again be satisfied which allows the possibility that

TABLE 3

Possible equilibria under assortative mating $(\beta=0) . \quad \hat{P}_{1}, \hat{P}_{2}$ and $\hat{P}_{3}$ are the equilibrium frequencies of $\mathrm{AA}, \mathrm{AA}^{\prime} \mathrm{A}^{\prime} \mathrm{A}^{\prime}$ respectively.

\begin{tabular}{ccccc}
\multicolumn{3}{c}{ Solution values } & \\
Solution & $\hat{\mathrm{P}}_{1}$ & $\hat{\mathrm{P}}_{2}$ & $\hat{\mathrm{P}}_{3}$ & Nature of equilibrium \\
$\mathrm{A}$ & 1 & 0 & 0 & Stable \\
$\mathrm{B}$ & 1 & 0 & $\frac{1}{2}$ & Unstable \\
$\mathrm{C}$ & 0 & 0 & 1 & Stable
\end{tabular}

there are additional solutions. With this restriction on $\bar{N}$, however, it can be shown that the two remaining equations in $\bar{N}$ (equation (5), table 2 and equation (8) cannot be satisfied for values of $\alpha, \hat{P}_{1}$ and $\hat{P}_{2}$ such that $0 \leqq \alpha, \hat{P}_{1}, \hat{P}_{2} \leqq 1$. In other words, the only biologically meaningful solutions to the equilibrium equations are those shown in table 3 .

The two trivial equilibria, $\hat{P}_{1}=1$ and $\hat{P}_{3}=1$, are stable whereas the equilibrium at $\hat{P}_{1}=\hat{P}_{3}=\frac{1}{2}$ is unstable; $P_{2}=0$ at all three equilibria. The nature of the equilibria (stable versus unstable) can be found by expanding equations (1) and (2) (table 2) in Taylor series about the equilibrium values and noting that a population would return to either $\hat{P}_{1}=1$ or $\hat{P}_{1}=0$ when perturbed slightly from these values, but that a perturbation from $\hat{P}_{1}=\frac{1}{2}$ initiates a series of ever increasing deviations from this equilibrium. The nature of the equilibria are also easily seen by numerical reiteration of equations (1) and (2). Fig. l illustrates two reiterative sequences covering 30 generations each. The upper curve was initiated with $P_{1}$ and $P_{2}$ equal to 0.50000001 and 0 respectively whereas the bottom curve shows a sequence initiated at $P_{1}=0.49999999$ and $P_{2}=0 \quad\left(\alpha=\frac{1}{2}\right.$ in both sequences). In other words, the sequences were started at the equilibrium at 0.5 plus or minus a perturbation of $10^{-8}$. The drive away from the equilibrium is nearly imperceptible for approximately 20 gener- 


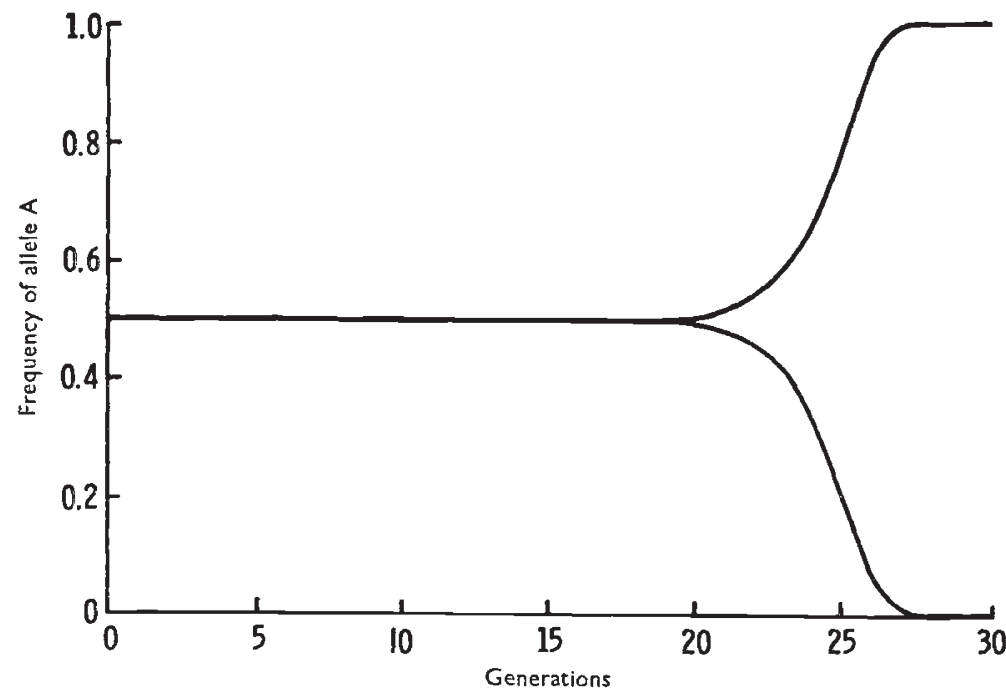

FIG. 1.-The " histories" of two populations perturbed slightly from the unstable equilibrium for assortative mating $\left(\alpha=\frac{1}{2}, \beta=0\right.$; initially, $P_{1}=0.50000001$ for the upper curve, $P_{1}=0.49999999$ for the lower curve, $P_{2}=0$ for both curves).

ations followed by a rapid elimination of the minority allele over the next 10 generations.

The dynamic aspects of a population under this scheme of assortative mating $(0 \leqq \alpha \leqq 1, \beta=0)$ were studied by numerical reiteration of equations (1) and (2). In general, the approach to either fixation or extinction of an allele is rapid.

Before considering the effects of mutation and selection, one additional case of assortative mating alone should be considered in the context of genetic polymorphisms at the assortative mating locus. This is the case when $\beta>0$ which models a locus that can cause assortative mating but does not have sufficient potency to effect total reproductive isolation. I have reiterated equations (1) and (2) for numerous combinations of $\alpha$ and

\section{TAble 4}

Incomplete assortative mating $(\beta>0) . \quad p$ is the frequency of the $\mathbf{A}$ allele; $\mathbf{P}_{1}, \mathbf{P}_{2}$ and $\mathbf{P}_{3}$ are the frequencies of genotypes $\mathrm{AA}, \mathrm{AA}^{\prime}$ and $\mathrm{A}^{\prime} \mathrm{A}^{\prime}$ respectively. Each population was initiated with $\mathrm{P}_{1}=0 \cdot 25$;

$P_{2}=0.51$ and reiterated until $p>0.99999$ or the number of generations exceeded 10,000

\begin{tabular}{|c|c|c|c|c|c|}
\hline \multirow[b]{2}{*}{$\alpha$} & \multirow[b]{2}{*}{$\beta$} & \multirow{2}{*}{$\begin{array}{c}\text { Number of generations } \\
\text { elapsed before } \\
p \geqslant 0.99999\end{array}$} & \multicolumn{3}{|c|}{$\begin{array}{l}\text { Population composition when } \\
\qquad p>0.99999\end{array}$} \\
\hline & & & $P_{1}$ & $P_{2}$ & $P_{\mathbf{3}}$ \\
\hline $0 \cdot 250$ & $0 \cdot 125$ & 17 & 0.99998 & 0.00002 & 0 \\
\hline 0.250 & $0 \cdot 250$ & 21 & 0.99999 & 0.00001 & 0 \\
\hline 0.500 & 0.250 & 34 & 0.99999 & 0.00001 & 0 \\
\hline 0.500 & 0.500 & 47 & 0.99999 & $0 \cdot 00001$ & 0 \\
\hline 0.750 & 0.375 & 70 & 0.99998 & $0 \cdot 00002$ & 0 \\
\hline 0.750 & 0.750 & 130 & 0.99998 & $0 \cdot 00002$ & 0 \\
\hline 1.000 & 0.500 & $10,000+$ & 0.99960 & $0 \cdot 00040$ & 0 \\
\hline 1.000 & 0.900 & $10,000+$ & 0.99789 & 0.00211 & 0 \\
\hline $1 \cdot 000$ & 0.950 & $10,000+$ & 0.99555 & $0 \cdot 00445$ & 0 \\
\hline $1 \cdot 000$ & 0.990 & $10,000+$ & 0.96460 & 0.03508 & 0.00032 \\
\hline
\end{tabular}


$\beta(0<\alpha \leqq 1, \beta \leqq \alpha)$, and a representative sample of these results is given in table 4. Each reiteration was started with $P_{1}=0.25$ and $P_{2}=0.51$. There appear to be no cases in this parameter set that result in a stable polymorphism for assortative mating genes other than the case where $\alpha=\beta=1$ which is equivalent to Hardy-Weinberg conditions. The rates of gene frequency change, however, are considerably slower as the amount of assortative mating is reduced (i.e. as $\alpha \rightarrow 1$ and $\beta \rightarrow 1$ ).

\section{Mutation at the assortative mating locus}

Suppose allele $A$ mutates to $A^{\prime}$ with probability $\nu$ and that the reverse mutation rate is the same. A fraction of each of the three classes of zygotes formed by assortative mating will be mutants. For example, a fraction, $\nu(1-\nu)$, of the $A A^{\prime}$ will have mutated to $A A$. To simplify the symbols used, let $\mu=\nu(1-\nu)$ be the probability that one allele in a diploid is a mutant while the other is not. The simultaneous occurrence of two mutations $\left(e . g . A A \rightarrow A^{\prime} A^{\prime}\right)$ would be rare, and these can be ignored without

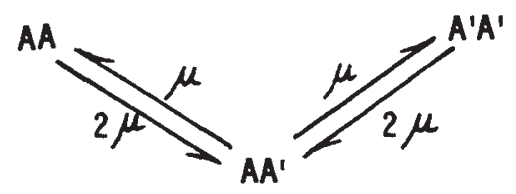

FIG. 2.-Transition probabilities between the three genotypes. $\mu=\nu(1-\nu)$ where $\nu$ is the per-allele mutation rate.

significantly altering the conclusions. The probabilities for mutations causing transitions among the three genotypes are illustrated in fig. 2.

In the absence of mutation, the frequencies of the three genotypes in the next generation are $P_{1}^{\prime}, P_{2}^{\prime}$ and $P_{3}^{\prime}$. With mutation:

$$
\begin{aligned}
& P_{1}^{\prime \prime}=(1-2 \mu) P_{1}^{\prime}+\mu P_{2}^{\prime} \\
& P_{2}^{\prime \prime}=(1-2 \mu) P_{2}^{\prime}+2 \mu P_{1}^{\prime}+2 \mu P_{3}^{\prime} \\
& P_{3}^{\prime \prime}=(1-2 \mu) P_{3}^{\prime}+\mu P_{2}^{\prime}
\end{aligned}
$$

where $P_{1}^{\prime \prime}, P_{2}^{\prime \prime}$ and $P_{3}^{\prime \prime}$ are the frequencies in the next generation of $A A, A A^{\prime}$ and $A^{\prime} A^{\prime}$ respectively.

I will explore the equilibrium conditions for the special case $\beta=0$. The appropriate difference equations are:

$$
\begin{aligned}
& \Delta P_{1}=P_{1}^{\prime \prime}-P_{1}=\left[(1-2 \mu) P_{1}^{\prime}+\right.\left.\mu P_{2}^{\prime}\right]-P_{1} \\
&= {\left[(1-2 \mu)\left(P_{1}^{2}+\alpha P_{1} P_{2}+\frac{1}{4} P_{2}^{2}\right) / \bar{N}\right.} \\
&\left.+\mu\left(\alpha P_{1} P_{2}+\frac{1}{2} P_{2}^{2}+\alpha P_{2} P_{3}\right) / \bar{N}\right]-P_{1} \\
& \Delta P_{2}=P_{2}^{\prime \prime}-P_{2}=\left[(1-2 \mu) P_{2}^{\prime}+\right.\left.2 \mu P_{1}^{\prime}+2 \mu P_{3}^{\prime}\right]-P_{2} \\
&=\left[(1-2 \mu)\left(\alpha P_{1} P_{2}+\frac{1}{2} P_{2}^{2}+\alpha P_{2} P_{3}\right) / \bar{N}+2 \mu\left(P_{1}^{2}+\alpha P_{1} P_{2}+\frac{1}{4} P_{2}^{2}\right) / \bar{N}\right. \\
&\left.+2 \mu\left(\frac{1}{4} P_{2}^{2}+\alpha P_{2} P_{3}+P_{3}^{2}\right) / \bar{N}\right]-P_{2}
\end{aligned}
$$


Equilibrium values can now be obtained by setting the right sides of equations (13) and (14) equal to zero and solving the resultant simultaneous algebraic equations for $P_{1}$ and $P_{2}$. The solutions are difficult to obtain, however, because the equations contain cubic terms. An additional assumption makes these equations linear; the taller then provide good approximations of the equilibrium frequencies specifically, if the secondorder terms involving $P_{1}$ and $P_{2}$ in equations (13) and (14) are assumed to be negligible, then the equations simplify to:

$$
\begin{aligned}
& \Delta P_{1}=\left(\alpha \mu P_{2}-P_{1} \bar{N}\right) / \bar{N} \\
& \Delta P_{2}=\left(2 \mu-4 \mu P_{1}-4 \mu P_{2}+\alpha P_{2}-P_{2} \bar{N}\right) / \bar{N}
\end{aligned}
$$

where

$$
\bar{N}=2 \alpha P_{2}-2 P_{1}-2 P_{2}+1 \text {. }
$$

These simplifications are justified by the fact that assortative mating alone will cause either $A$ or $A^{\prime}$ to be eliminated from the population. With the addition of mutation, the "extinct" allele would occur at a low frequency. For example, if $A^{\prime}$ were the predominant allele, $P_{3}$ would be near unity, $P_{1}$ and $P_{2}$ would be small, and the squares and cross-products of $P_{1}$ and $P_{2}$ would be negligibly small. Setting the simplified difference equations ((15) and (16)) equal to zero and solving yields:

$$
\begin{aligned}
& \hat{P}_{1}=\alpha \mu \widehat{P}_{2} \\
& \hat{P}_{2}=2 \mu /\left(4 \alpha \mu^{2}+4 \mu-\alpha+1\right)
\end{aligned}
$$

Equations (18) and (19) give approximate equilibrium frequencies for genotypes $A A$ and $A A^{\prime}$, respectively, when assortative mating is counteracted by mutation.

Thus, when there is mutation at a locus that causes assortative mating and the alternate alleles are adaptively equivalent, an equilibrium will be established wherein one allele will be held at a low frequency by assortative mating. This is in contrast to a locus that does not cause assortative mating; there, new adaptively neutral mutations could rise to high frequencies in the population or even become fixed depending on the magnitude of the reverse mutation rate. The equilibrium frequencies of genotype $A A^{\prime}$ are given in table 5 for several values of $\mu$ and $\alpha$. Since I assumed that the mutation rates from $A$ and $A^{\prime}$ and vice versa were identical, $\hat{P}_{2}$ would be the same regardless of which allele predominated. However, $\hat{P}_{1}$ and $\hat{P}_{3}$ would depend on which allele was initially most frequent; if allele $A$ was predominant initially, then the population would come to equilibrium with $\hat{P}_{1}$ near unity. Table 5 gives approximate values of $\hat{P}_{2}$ as determined from equation (19) along with exact values determined by apparent convergence to equilibrium of numerical reiterations of the difference equations for the combined effects of mutation and assortative mating (these equations are not given explicitly in this article). The accuracy of equation (19) can be assessed by comparing columns 3 and 4 ; the approximate values are very close for reasonable values of $\mu$ and for all values of $\alpha$ except those very close to 1 . 


\section{TABLE 5}

Equilibrium frequency of genotye $\mathrm{AA}^{\prime}$ when mutation occurs at an assortative mating locus. $\hat{\mathrm{P}}_{2}$ is the equilibrium frequency of $\mathrm{AA}^{\prime}, \mu=\nu(1-\nu)$ where $\nu$ is the per-allelle mutation rate and $\alpha$ the assortative mating coefficient $(\beta=0)$. See text for full explanation

$\begin{array}{ccrc}\mu & \hat{\mathrm{P}}_{2} \text { determined by } & \begin{array}{c}\hat{\mathrm{P}}_{2} \text { determined by } \\ \text { equation }(19)\end{array} & \begin{array}{c}\text { numerical reiteration } \\ \mu\end{array} \\ 1 \times 10^{-6} & 0.5 & 4.00 \times 10^{-6} & 4.00 \times 10^{-6} \\ 5 \times 10^{-6} & 0.5 & 2.000 \times 10^{-5} & 2.000 \times 10^{-5} \\ 1 \times 10^{-5} & 0.5 & 4.000 \times 10^{-5} & 4.000 \times 10^{-5} \\ 1 \times 10^{-5} & 0.75 & 7.999 \times 10^{-5} & 7.999 \times 10^{-5} \\ 1 \times 10^{-5} & 0.9 & 1.9992 \times 10^{-4} & 1.9984 \times 10^{-4} \\ 1 \times 10^{-5} & 0.95 & 3.9968 \times 10^{-4} & 3.9857 \times 10^{-4} \\ 1 \times 10^{-5} & 1.0 & 4.9999500 \times 10^{-1} & 6.30113 \times 10^{-3} \\ 1 \times 10^{-4} & 0.5 & 3.9968 \times 10^{-4} & 4.0000 \times 10^{-4} \\ 1 \times 10^{-3} & 0.5 & 3.96824 \times 10^{-3} & 3.99998 \times 10^{-3} \\ 1 \times 10^{-2} & 0.5 & 3.702332 \times 10^{-2} & 3.998300 \times 10^{-2}\end{array}$

\section{Selection at the assortative mating locus}

I will address two questions regarding selection acting upon the assortative mating locus. (1) Are there selection regimes that, in conjunction with assortative mating, lead to allelic polymorphism and (2) how much selection is necessary to counteract the "centrifugal" force of assortative mating? Considering various selection regimes and values of assortative mating parameters, an infinity of cases could be considered. However, a few numerical examples suffice to answer these questions.

Equations for the frequencies of the three genotypes in the next generation in terms of their present frequencies, when both assortative mating and selection are considered, can be formulated from table 6. Here $P_{1}^{\prime}, P_{2}^{\prime}$ and $P_{3}^{\prime}$ are the frequencies of the zygotes at fertilisation after assortative mating and mutation (if $\mu>0$ ) have occurred. Between zygote formation and adulthood, selection will adjust these frequencies to those given on line 4 of table 6 . The survivors that comprise these classes will then contribute to the next generation as prescribed by the assortative mating scheme. $P_{1}^{\prime}$, $P_{2}^{\prime}$ and $P_{3}^{\prime}$ can be substituted by the functions of $P_{1}, P_{2}$ and $P_{3}$ developed earlier to yield the desired recurrence equations.

First consider the question: How much selection is necessary to overcome the "centrifugal " force of assortative mating? One way to approach this problem is to consider a population in which $A^{\prime}$ is initially fixed. Suppose $A$ has entered the population by mutation or migration and the

\section{TABLE 6}

Model for selection at the assortative mating locus. $\mathrm{P}_{1}^{\prime}, \mathrm{P}_{2}^{\prime}$ and $\mathrm{P}_{3}^{\prime}$ are the frequencies of the respective genotypes after assortative mating and mutation (if $\mu>0$ ) have occurred

\begin{tabular}{lccc} 
Genotype & $A A$ & $A A^{\prime}$ & $A^{\prime} A^{\prime}$ \\
$\begin{array}{l}\text { Frequency of zygotes at } \\
\text { fertilisation }\end{array}$ & $P_{1}^{\prime}$ & $P_{2}^{\prime}$ & $P_{3}^{\prime}$ \\
Fitness & $W_{A A}$ & $W_{A A^{\prime}}$ & $W_{A^{\prime} A^{\prime}}$ \\
\cline { 2 - 3 } & $P_{1}^{\prime} W_{A A} / \bar{W}$ & $P_{2}^{\prime} W_{A A^{\prime}} / \bar{W}$ & $P_{3}^{\prime} W_{A^{\prime} A^{\prime} / \bar{W}}$
\end{tabular}
where: $\bar{W}=P_{1}^{\prime} W_{A A}+P_{2}^{\prime} W_{A A^{\prime}}+P_{3}^{\prime} W_{A_{A}^{\prime}}$ 
two genotypes containing $A\left(A A\right.$ and $\left.A A^{\prime}\right)$ are favoured in a pattern of directional selection; i.e. $W_{A A} \geqq W_{A A^{\prime}}>W_{A^{\prime} A^{\prime}}$ or $W_{A A}>W_{A A^{\prime}} \geqq W_{A^{\prime} A^{\prime}}$, where $W_{A A}$, etc. are the respective genotypic fitnesses. In the absence of assortative mating, $A$ would increase to fixation, but, when the alleles in question also cause assortative mating, the relative adaptive values of the invading genotypes must exceed certain minimum values if the invading allele is to increase in frequency. It is difficult to state what are the minima; they depend upon $\alpha, \beta, W_{A A}, W_{A A^{\prime}}$ and $W_{A^{\prime} A^{\prime}}$ as well as the initial frequencies of the novel genotypes. Five illustrative examples are given in table 7; in all these examples $\alpha=0.5, \beta=0$, and the initial genotypic frequencies were $P_{1}=0, P_{2}=0.00004$. Values of $W_{A A}$ and $W_{A A^{\prime}}$ were specified and the corresponding threshold value of $W_{A^{\prime} A^{\prime}}$ determined by trial-and-error reiteration of the recurrence equations. (Note that all fitnesses are related to $W_{A A}=1$ in these examples.) Values of $W_{A^{\prime} A^{\prime}}$

TABLE 7

Selection regimes for which an invading gene (A) will increase in frequency

\begin{tabular}{|c|c|c|c|c|}
\hline & & & \multicolumn{2}{|c|}{$\begin{array}{c}\text { Allele } A \text { will increase for all } \\
\text { values of } X \text { less than: }\end{array}$} \\
\hline \multicolumn{3}{|c|}{ Selection regime } & without & with \\
\hline$W_{A A}$ & $W_{A A^{\prime}}$ & $W_{A^{\prime} A^{\prime}}$ & $(\mu=0)$ & $\left(\mu=10^{-5}\right)$ \\
\hline 1 & 1 & $X$ & 0.500 & 0.504 \\
\hline 1 & 0.75 & $X$ & 0.375 & 0.378 \\
\hline 1 & 0.5 & $X$ & 0.250 & $0 \cdot 252$ \\
\hline 1 & 0.25 & $X$ & $0 \cdot 125$ & $0 \cdot 126$ \\
\hline$i$ & $X$ & $X$ & 0.00002 & - \\
\hline
\end{tabular}

exceeding the threshold lead to elimination of the invading gene; values of $W_{A^{\prime} A^{\prime}}$ less than the threshold lead to fixation of the invading gene. Thus, for example, when $W_{A A}=1$ and $W_{A A^{\prime}}=0.5, A$ cannot invade unless $W_{A^{\prime} A^{\prime}}$ is less than approximately $0 \cdot 250$.

Inspection of table 7 reveals that the invading gene must enhance the relative fitnesses of its genotypes rather substantially if it is to overcome the disadvantage of being rare in an assortatively mating population. Line 1 of table 7 represents the case where the invading allele is dominant with regard to fitness; line 5 is the case where it is recessive. The precision of the determined values in table 7 is limited to three decimal places, and the fact that the first four values round off to precisely one-half of the corresponding heterozygote fitness should not be misconstrued-in each case it was clear that the actual threshold was slightly greater than half of $W_{A A^{\prime}}$.

Returning now to the question of genetic polymorphism, when selection occurs at the assortative mating locus, a necessary condition for polymorphism apparently is identical to that of selection acting alone; viz. the heterozygote must be more fit than either homozygote. The conclusion is not rigorous because it was arrived at by numerical analysis, but I have been unable to find any other situation resulting in polymorphism. On the other hand, heterozygote advantage is not a sufficient condition. The actual values of $W_{A A}, W_{A A^{\prime}}$ and $W_{A^{\prime} A^{\prime}}$ as well as $\alpha, \beta$ and the initial values of $P_{1}$ and $P_{2}$ all determine whether or not a given population will evolve to an internal equilibrium. 
Two sets of numerical examples illustrate some of the salient features of the interaction between assortative mating and stabilising selection. The first example addresses the question: How intense must selection be to lift a rare allele to a higher equilibrium frequency. $W_{A A^{\prime}}$ was set at 1.0 and successive symmetrical sets of homozygous fitnesses $\left(W_{A A}=W_{A^{\prime} A^{\prime}}\right)$ were tried until the threshold was found (to the nearest 0.001 ) at which the rare allele overcame the counteracting force of assortative mating and increased in frequency to equilibrium. The initial genotypic frequencies were $P_{1}=0.99996, P_{2}=0.00004 ; \alpha=0.5, \beta=0$. The threshold was found to be in very slight excess of 0.500 ; i.e. if $W_{A A}=W_{A^{\prime} A^{\prime}}<0.500$ the frequency of the rare allele will increase to the internal equilibrium. So, again, selection must be rather intense to overcome the force of assortative mating acting against the rare allele. It is also apparent that assortative mating reduces the number of heterozygotes. In the absence of assortative mating, the equilibrium frequencies for the selection regime

$$
W_{A A}=W_{A^{\prime} A^{\prime}}=0.5, \quad W_{A A^{\prime}}=1
$$

are $\hat{P}_{1}=\hat{P}_{3}=0 \cdot 167, \hat{P}_{2}=0.667$; with assortative mating the respective frequencies are $0 \cdot 191$ and $0 \cdot 618$.

The second set of examples concerns the situation where there is overdominance $\left(W_{A A}<W_{A A^{\prime}}=1>W_{A^{\prime} A^{\prime}}\right)$ but there is an asymmetry in homozygote fitnesses (i.e. $\left.W_{A A} \neq W_{A^{\prime} A^{\prime}}\right)$. The "histories" of two populations experiencing this pattern of selection are graphed in fig. 3. The specific selection regimes were $\left[W_{A A}=0.4, W_{A A^{\prime}}=1, W_{A^{\prime} A^{\prime}}=0.6\right]$ and $\left[W_{A A}=0.3, W_{A A^{\prime}}=1, W_{A^{\prime} A^{\prime}}=0.7\right]$; in both examples $\alpha=0.5, \beta=0$, and $P_{1}=0.95, P_{2}=0.05$ at the onset. For comparison, the "histories" of "control" populations are also plotted-these differ only in that there

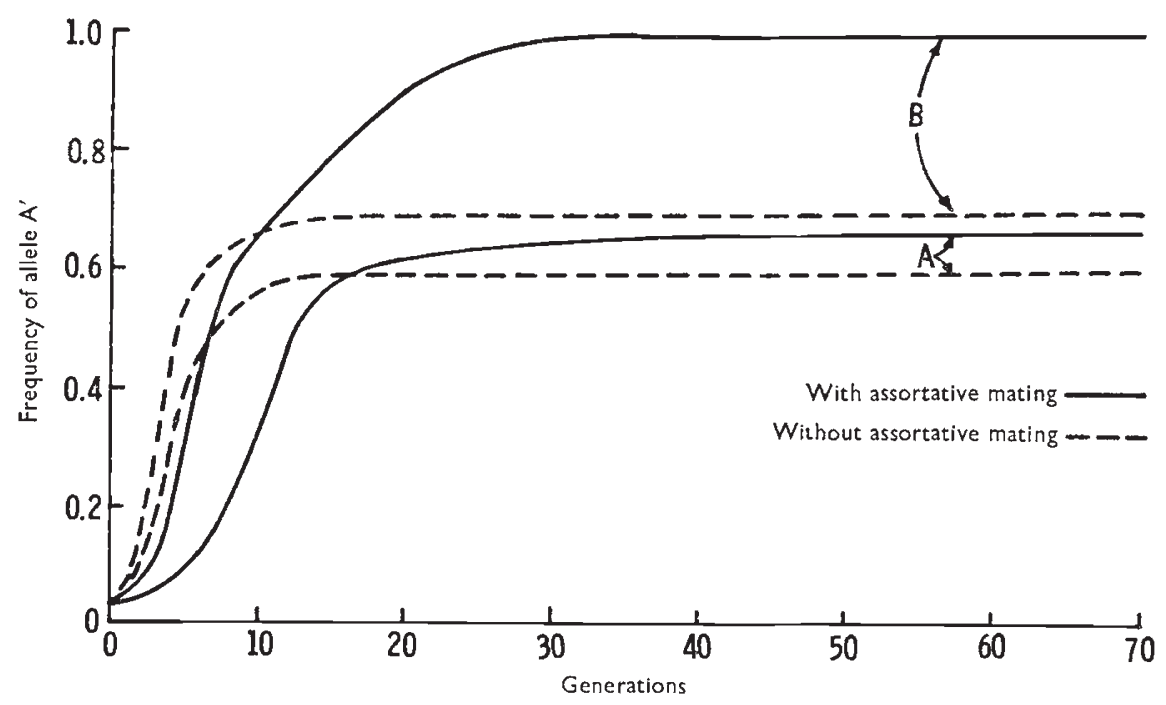

Fig. 3.-Selection favouring the heterozygote at the assortative mating locus. The selection regime for the pair of curves labelled $A$ is $W_{A A}=0.4, W_{A A^{\prime}}=1, W_{A^{\prime} A^{\prime}}=0.6$; for pair $B$ : $W_{A A}=0.3, W_{A A^{\prime}}=1, W_{A^{\prime} A^{\prime}}=0.7$. The initial genotypic frequencies are $P_{1}=0.95$, $P_{2}=0 \cdot 05$. The dashed curve indicates selection only; the solid curve is selection plus assortative mating. For the assortative mating simulations, $\alpha=\frac{1}{2}, \beta=0$. 
is no assortative mating. It can be seen in fig. 3 that when there is overdominance but an asymmetry in the selection pattern, that assortative mating shifts the equilibrium towards fixation of the favoured allele. As the asymmetry is accentuated, however, a point is reached at which selection is overpowered and assortative mating drives the equilibrium to the fixation point.

\section{Mutation and SElegtion at the assortative mating locus}

It is plausible that recurring mutation in conjunction with selection might lower the threshold at which a new mutant could increase in frequency. While this is true in a strict sense, the threshold change is very slight. This conclusion is based on a series of reiterations that was identical to the directional selection series presented in table 7 except $\mu=0$ in the former and $\mu=10^{-5}$ in the latter. These results are also tabulated in table 7. Thus, for example, when $W_{A A}=1$ and $W_{A A^{\prime}}=0.5(\alpha=0.5$, $\beta=0$ ) allele $A$ could invade the population if $W_{A^{\prime} A^{\prime}}<0.250$ without recurring mutation and for $W_{A^{\prime} A^{\prime}}<0 \cdot 252$ with mutation.

\section{Discussion}

The mass-action formulation clearly indicates that an assortative mating locus is not likely to be polymorphic. Assortative mating, by itself, eliminates the rarer allele from the population. If the alleles are pleiotropic in the sense that they not only cause assortative mating but affect fitness in some additional way, then the locus will be polymorphic provided that the heterozygote is substantially more fit than the homozygotes. However, assortative mating will prohibit the flux of adaptively neutral assortative mating mutations through the population and even inhibit the influx of weakly to moderately adaptive mutations.

The essential feature of the mass-action formulation is that there is a cost associated with assortative mating. Stated simply, an organism with an odd genotype is less likely to find a mate. In sharp contrast, there is no cost implicit in the assortative mating scheme most often used in theoretical studies where speciation is supposed to result from the evolution of premating reproductive isolation. The most commonly used model of assortative mating was originally formulated by O'Donald (1960). He proposed a one-locus-two-allele model where individuals comprising a constant fraction of the population mate assortatively while the remaining individuals mate randomly. In this scheme, all individuals mate, it is a question of whether they mate with their own phenotype or one drawn at random. A resultant peculiarity of the constant-fraction model is that allelic frequencies remain constant from generation to generation, whereas the minority allele evolves to extinction according to the mass-action model.

Is it realistic to assume that there is a cost associated with assortative mating? For certain kinds of mating systems it clearly is; e.g. crosspollination in flowering plants suggests a number of cases. The correlation of inflorescence statures in mating pairs of Lythrum salicaria is one example. This is the direct result of the tendency of hive-bees to forage in a horizontal plane. Insect pollinators exhibit another behaviour that results in assortative mating among flowering plants; viz. flower constancy. Flower 
constancy is the tendency for insects to work a single kind of flower at a time. This phenomenon is particularly well developed and well documented in bees (see Grant, 1950; Free, 1970; and Proctor and Yeo, 1973 for reviews and references). Hive-bees, for example, forage on a wide range of species, but individual workers usually visit only a single species on each foraging trip. Further, flower fidelity is retained over a period of several days (Free, 1970). Mather (1947) showed that flower constancy in bees was an effective isolating mechanism between two species of snapdragon (Antirrhinum majus and A. glutinosum) grown in experimental plots; aside from assortative mating effected by bees, these species are fully interfertile. In a similar set of experiments Grant (1949) showed the efficacy of hive-bee flower constancy in preventing miscegenation among subspecies of Gilia capitata (polemoniaceae). Levin (1972) demonstrated that minority corolla variants were at a disadvantage in mixed populations of Phlox drummondii and concluded: "We cannot discount the possibility that, as a rule, frequency-dependent selection for genotypes governing floral signals is concomitant with a minority disadvantage". Discrimination by bees is apparently based on floral geometry and/or scent, both of which are obviously under genetic control. Thus, an odd floral type that falls outside the acceptable search "image" of a specific pollinator will be selected against and the allele(s) that caused the aberrant phenotype will decrease in frequency. The mass-action model predicts that characteristics of this nature should have a relatively low heritability in natural populations.

Flowering time could also effect assortative mating. A change in flowering season could result in reproductive isolation as could a change in the cycle of a flower that opens and closes daily. A possible example of the latter was reported by Raven (1961) in two species of Oenothera. Oe. clavaeformis and $O e$. brevipes occur in the south-western United States where they occasionally hybridise whenever found in mixed populations. Flowers of Oc. brevipes open approximately 2 hours before sunrise, whereas flowers of Oc. clavaeformis open about 2 hours before sunset. As a consequence, the two species are largely pollinated by distinct species of bees, and this difference in diurnal flowering time appears to be a major component of the complex of mechanisms isolating the two species. Again, the odd plant that flowers out of phase will have a reduced chance for cross-pollination and will be selected against unless this loss is compensated by a fitness gain in some other respect.

Whether a cost would be associated with assortative mating in more complex systems can be argued. Perhaps the most general case in higher animals is one in which sexes are separate and females produce gametes at a relatively high cost. Accordingly, females are choosy whereas males are promiscuous and decidedly less fastidious. This is roughly the situation in Drosophila. One might argue that all females are mated in this situation, and, therefore, they have nothing to lose in being choosy. However, if courtship and discrimination take time, and if females are exposed to death risk during this time, females that are unnecessarily choosy will be selected against. In the economy of nature it is doubtful that an individual could ever afford to delay reproduction because of fastidiousness unless this habit brought a compensatory dividend in fitness.

Conclusions deduced from the constant-fraction and mass-action models are quite disparate: an assortative mating locus that satisfied the essential 
assumptions of the constant-fraction model would be polymorphic and apparently would respond to an indirect selection pressure resulting from disruptive selection at a second locus (Endler, 1977; Caisse and Antonovics, 1978). An assortative mating locus that satisfied the essential assumptions of the mass-action model, in contrast, would be polymorphic only to an extent allowed by recurring mutation and may or may not respond to the same selection regime depending on the intensity of hybrid inferiority. Which of these conclusions is most likely true depends on which model most realistically portrays natural assortative mating processes. It is implicit in the constant-fraction formulation that all individuals mate, and that there is no determination of fitness associated with this event. Although assortative mating mechanisms need to be studied on a case-by-case basis, this seems to be a generally implausible assumption where speciation processes are of interest. Further, assortative mating schemes that do result in polymorphisms appear to be the exception rather than the rule. Scudo and Karlin (1969) asserted as a "robust" conclusion that assortative mating at an autosomal locus with dominance will never produce a stable polymorphism. In the several cases they studied where there was incomplete dominance (Karlin and Scudo, 1969), only two resulted in stable polymorphisms. In both cases, the necessary conditions are analogous to those of natural selection causing polymorphism; viz. the intensity of assortment in the heterozygote has to be greater than that in the homozygotes.

The actual extent of genic polymorphism at loci with the potential of causing assortative mating needs further investigation. Obviously, a great deal of caution should be exercised in extrapolating inferences about speciation from a one-locus-two-allele model of assortative mating, but, on the other hand, inferences based on the assumption that variation for assortative mating genes (if there is any) will respond to selection favouring reproductive isolation are at least as suspect.

Acknowledgments.--I would like to thank Gordon Hines, John Maynard Smith and Michael Rose for critically reading a rough draft of this paper. I would like to especially thank Gordon Hines who suggested some of the mathematical approaches used in this paper. This work was done at the University of Sussex under the sponsorship of a NATO Postdoctoral Fellowship in Science. Part of the work was supported by National Science Foundation Grant No. DEB 77-15352.

\section{REFERENCES}

GAISsE, M., AND ANTONovics, J. 1978. Evolution in closely adjacent plant populations. IX. Evolution of reproductive isolation in clinal populations. Heredity, 40, 371-384. довzнANsкy, тн. 1940. Speciation as a stage in evolutionary divergence. Amer. Nat., 74, 97-127.

ENDler, J. A. 1977. Geographic Variation, Speciation, and Clines. Princeton Univ. Press, Princeton, N.J.

FREE, J. B. 1970. Insect Pollination of Crops. Academic Press, London.

GRANT, v. 1949. Pollination systems as isolating mechanisms in flowering plants. Evolution, $3,82-97$.

GRANT, v. 1950. The flower constancy of bees. Botanical Review, 16, 379-398.

HUXLEY, J. S. 1942. Evolution: The Modern Synthesis. Allen and Unwin, London.

KARLIN, s., AND scudo, F. M. 1969. Assortative mating based on phenotype: II. Two autosomal alleles without dominance. Genetics, 63, 499-510.

LEVIN, D. A. 1972. Low frequency disadvantage in the exploitation of pollinators by corolla variants in Phlox. Amer. Nat., 106, 453-460.

LEVIN, D. A., AND KERSTER, H. W. 1973. Assortative pollination for stature in Lythrum salicaria. Evolution, 27, 144-152. 
MAYR, E. 1963. Animal species and Evolution. Belknap Press, Cambridge, Mass.

MATHER, K. 1947. Species crosses in Antirrhinum I. Genetic isolation of the species majus, glutinosum and orontium. Heredity, 1, 175-186.

o'DONALD, P. 1960. Assortative mating in a population in which two alleles are segregating. Heredity, 15, 389-396.

PROCTOR, M., AND YEO, P. 1973. The Pollination of Flowers. Collins, London.

RAVEN, P. H. 1961. Interspecific hybridization as an evolutionary stimulus in Oenothera. Proc. Linnean Soc. London, 1973, 92-98.

REMINGTON, C. L. 1968. Suture-zones of hybrid interaction between recently joined biotas. Evolutionary Biology, 2, 321-428.

scuno, R. M., AND KARLIN, s. 1969. Assortative mating based on phenotype, I. Two alleles with dominance. Genetics, 63, 479-498. 